\title{
Iron losses of a linear generator for a hybrid vehicle concept
}

\author{
F. Rinderknecht \\ Institute of Vehicle Concepts \\ German Aerospace Center \\ Stuttgart, Germany \\ frank.rinderknecht@dlr.de
}

\begin{abstract}
This publication deals with the calculation and the measurement of the iron losses in a linear generator. The linear generator is intended for use in a hybrid power train design for a vehicle. The system surrounding the linear generator and some parameters have an influence on the linear generator and its iron losses. They will be explained to achieve a fundamental understanding of the requirements and the resulting topology selection. Furthermore, the question if the iron losses from a linear generator can be calculated analytically and how exact they can be calculated will be discussed. The calculated values are compared with the values measured on a prototype to permit an assessment of the quality of the calculations.
\end{abstract}

Keywords-component; Alternators; iron losses; free-piston linear generator; linear motors; linear generators; permanent magnet generators; synchronous generators

\section{INTRODUCTION}

Efficiency improvements in order to reduce the production of $\mathrm{CO}_{2}$ are required in all technical areas of life to limit the anticipated climate changes [1] to an extent sustainable for humanity. Current approaches in the field of transportation cover both the increasing of efficiency of conventional drives and the development of hybrid, battery, fuel cell and range extender concepts, and combinations of these. The aim of every alternative power train design is to transform the stored energy into a useful form of energy with the greatest possible efficiency.

This requirement is met particularly well by a free piston linear generator. It is capable of transforming chemical energy into electrical energy by means of a combustion process. As explained in [2], the high degree of efficiency and its independence of the load level are inherent in the design of the free-piston linear generator. This is achieved by keeping the system frequency constant and adapting to the power demanded by variation of the stroke and compression ratio. Furthermore, these system characteristics mean that the freepiston linear generator can be operated with both conventional fuels such as petrol, diesel and gas and with alternative fuels such as sun fuel, synthetic fuel, hydrogen etc. In the development of scenarios for the introduction of alternative power train concepts the free-piston linear generator can be seen as a bridging technology between conventional power train technologies and fuel cell technology. Particularly since the free-piston linear generator supplies electric current at its energy interface as fuel cell do.

The linear generator discussed in this paper is a component in the free-piston linear generator with the task of transforming mechanical energy into electrical energy.

\section{FREE-PISTON LINEAR GENERATOR SYSTEM}

The concept of the free-piston linear generator will be described in this section in order to assist in the understanding of the boundary conditions affecting the linear generator.

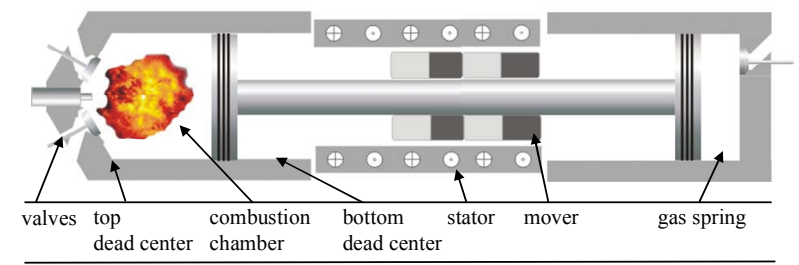

Figure 1. Illustration of the principle of the free-piston linear generator

\section{A. Construction}

The free-piston linear generator in Fig. 1 is based on a double piston system (hatched) built into a cylinder. At one end there is the combustion chamber for the combustion of a liquid or gaseous energy carrier. The gas exchange of the combustion chamber is controlled by electrically actuated valves in the cylinder head. An adjustable gas spring is built into the end opposite the combustion chamber. The gas mass in the gas spring can be adjusted by means of a control valve. This allows regulation of the spring constant of the gas spring. The mover is mounted between the piston of the combustion chamber and the piston of the gas spring. Permanent magnets are glued to the mover and additionally secured by a fiberglass reinforced plastic bandage. The mover with the permanent magnets is enclosed by a stator with integrated generator winding and the necessary cooling system necessary. The mover and the stator together make up the linear generator discussed in this paper. 


\section{B. Operation}

At the start of the load cycle the double piston is at the top dead center (TDC) and a flammable gas mixture previously introduced into the combustion chamber is ignited by a spark plug. The double piston with its integrated mover begins to move towards the gas spring (bottom dead center). The movement of the magnets induces a voltage in the coils of the stator which drives a corresponding electric current. Half of the mechanical energy released in the combustion is captured between top dead center and bottom dead center in this way. The other half is stored in the gas spring and extracted by the linear generator on the return stroke of the double piston (BDC-TDC). The gas in the combustion chamber is actively flushed while the piston is close to the bottom dead center, by introducing fresh air. When the fresh gas is compressed and the double piston has arrived at TDC, the next load cycle can begin. The variation of the output power is achieved by adjustment of the stroke, which is determined by the gas mass in the gas spring. The mechanical frequency of the double piston system is kept constant.

\section{Boundary conditions}

\section{1) Force diagram}

Fig. 2 shows, by way of example, one of the force graphs specified in [3]. The force shows the same development at the other operating points. Only the amplitude is varied. A requirement that is deduced from Fig. 2 is the need to be able to decouple a constant force over the complete stroke with the linear generator is. A short and a long stator design arise from this requirement as the possible design principles for the linear generator.

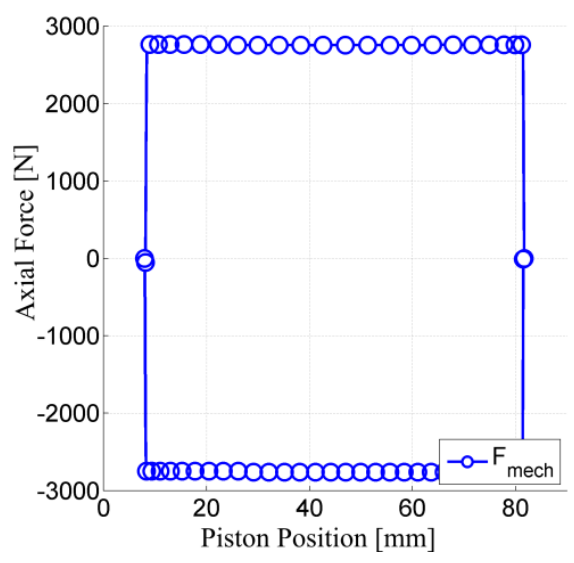

Figure 2. Force curve required in [3]

This requirement must, however, take into account the fact that the weight of the mover must be kept as low as possible for kinematic reasons. This is the case if the length of the mover is selected in such order that the maximum required force can be decoupled by all the magnets which are covered by the stator coils.

In the case of a short stator arrangement, which meets the requirement explained above, a portion of the magnets in the mover exits the stator and thus no longer contributes to the conversion of power. This results in a decrease of the electrical power output. To counteract the drop in force the current has to be increased which results in higher electrical losses and reduces the efficiency of the linear generator.

In the case of the long stator arrangement, the mover is permanently inside the stator, which leads to a constant force given a constant current. In average the current necessary to generate a constant force is lower in the long stator arrangement than in the short stator arrangement. As result there are less copper losses and the long stator arrangement (see Fig. 3) has a better degree of efficiency. Therefore the arrangement is used in the free-piston linear generator.

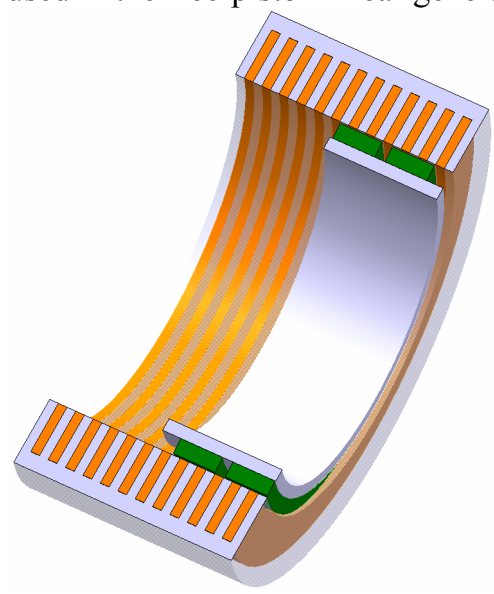

Figure 3. Shortest possible long stator design for the linear generator

\section{2) Production costs}

To keep the cost down, the development of the linear generator must aim for a low number of different parts, modular construction, a reduction of the complexity of manufacturing the components and a design for the parts that is susceptible to automated manufacturing. These requirements are expressed in the selection of a waste-free sheet metal cut-out. If the teeth and the slots are given the same width, as illustrated in Fig. 4, the part can be cut from sheet metal with practically no waste.

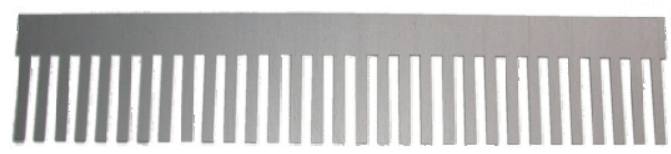

Figure 4. Practically waste-free sheet metal cut-out with identical width of tooth and slot

One result of this slot geometry is that there are a lot of slot harmonics in the system. They produce some iron losses in the stator. These kinds of iron losses are conventionally not considered. 


\section{3) Movement}

Another source of iron losses in this kind of generator is the movement distance. The length of the movement can be changed in this application. The result of this working characteristic is that the flux density does not have complete sine wave-form. So the standard way of the iron losses calculation cannot be done. The induced harmonics have to be considered.

In the following chapters the analytical calculation method of the iron losses under consideration of the special boundary conditions is represented.

\section{CALCULATION OF THE IRON LOSSES OF LINEAR GENERATOR}

\section{A. The principle of the iron losses calculation}

The iron losses $\mathrm{P}_{\text {lfe }}$ are a sum of the losses based on the hysteresis losses $\mathrm{P}_{\mathrm{lfe}, \mathrm{Hy}}$ and the eddy-current losses $\mathrm{P}_{\mathrm{lfe}, \mathrm{Wi}}$ as shown in (1).

$$
P_{l f e}=P_{l f e, H y}+P_{l f e, W i}
$$

The hysteresis losses can be calculated with the relationship from [4] and [10].

$$
P_{l f e, H y}=m \cdot v_{15 H}\left(\frac{f}{50 H z}\right)\left(\frac{B}{1.5 T}\right)^{2}
$$

In which $\mathrm{m}$ is the mass of the iron material, $v_{15 \mathrm{H}}$ is the material constant indicated from the manufacturer of the material, $\mathrm{f}$ is the frequency of the field that creates the losses and $\mathrm{B}$ is the flux density.

The eddy-current losses can be determined by

$$
P_{l f e, W i}=m \cdot \sigma_{15 H}\left(\frac{f}{50 H z}\right)^{2}\left(\frac{B}{1.5 T}\right)^{2}
$$

To consider the effect of the harmonic waves in the flux density it is important to introduce the factor $\kappa$ which is explained in [6].

$$
k=\frac{\sum\left(\lambda \hat{B}_{\lambda}\right)^{2}}{B_{\max }^{2}}
$$

The number of the harmonic is $v$. By using this factor (1) becomes

$$
P_{l f e}=\kappa \cdot P_{l f e, W i}+P_{l f e, H y}
$$

To get a precise result it is important to know the harmonic based on the effects of the flux density $\mathrm{Bv}$. The cause for the harmonics is explained in the chapter boundary conditions.

\section{B. Analytical calculation of the $B$ field}

To calculate the flux density and the included harmonics of the Stator Bs and the flux density of the mover the flux density of each component has to be developed in a Fourier series. After this both field components have to be superimposed. The result is a flux density which is the base of the iron losses including the first harmonic and higher harmonics.

\section{1) Stator field}

The flux density Bs induced by the stator can be calculated with (6).

$$
B_{s}(x, t)=\frac{\mu_{0}}{\delta} \cdot \int a(x, t) d l
$$

The electrical loading $\mathrm{a}(\mathrm{x}, \mathrm{t})$ may be determined from the geometry in Fig. 5 with a current in slot $2 i_{n 2}$. These coils are geometrically offset by $2 \pi / 3$. This corresponds to an angle of $120^{\circ}$ in a rotating machine.

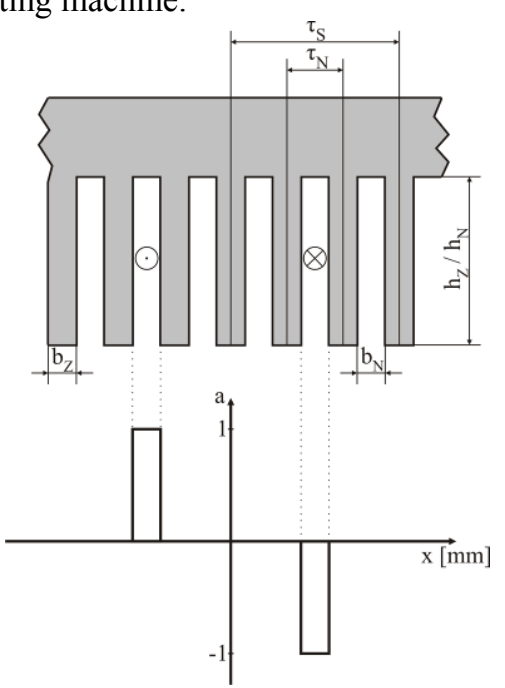

Figure 5. Geometry for the Fourier series approach for the calculation of the stator electrical loading

The electrical loading results from these assumptions through a Fourier series approach to yield (7). 


$$
\begin{aligned}
a(x)=\frac{4}{\pi} \cdot \frac{\omega_{n} \cdot i_{n 2}}{b_{N}} \\
\cdot \sum_{v=0}^{\infty}\left[\begin{array}{l}
\frac{1}{2 v+1} \cdot \cos \left((2 v+1) \pi\left(\frac{1}{2}-\frac{b_{n}}{2 \tau_{s}}\right)\right) \\
{\left[\begin{array}{l}
\sin \left((2 v+1)\left(\frac{\pi}{\tau_{s}}(x)\right)\right) \\
+\sin \left((2 v+1)\left(\frac{\pi}{\tau_{s}}(x)-\frac{2}{3} \pi\right)\right) \\
+\sin \left((2 v+1)\left(\frac{\pi}{\tau_{s}}(x)+\frac{2}{3} \pi\right)\right)
\end{array}\right]}
\end{array}\right]
\end{aligned}
$$

If this approach is transferred to a three-phase stator current system with $2 \pi / 3$ phase-shifted currents, (8) concludes after a few transformations.

$$
a(x, t)=\frac{3 \cdot \omega_{n}}{\tau_{s}} I_{1} \sqrt{2} \sum_{v=0}^{ \pm \infty} \xi_{n(v)} \xi_{s(v)} \cdot \sin \left(v \frac{\pi}{\tau_{s}} x-\omega t\right)
$$

In this equation $\omega_{\mathrm{n}}$ is the number of windings per coil and $\omega$ is the frequency of the current. The slot width factor $\xi_{n}$ according to [6] is pictured in (9).

$$
\xi_{n}(v)=\frac{\sin \left(\frac{1}{6} v \pi \frac{b_{N}}{\tau_{N}}\right)}{\frac{1}{6} v \pi \frac{b_{N}}{\tau_{N}}}
$$
(10)

The pitch factor $\xi_{\mathrm{s}}$ with the coil width W [6] is described in

$$
\xi_{s}(v)=\sin \left(v \frac{\pi}{2} \frac{W}{\tau_{P}}\right)
$$

The result of including (8) in (6) is shown in (11).

$$
\begin{aligned}
B_{s}(x, t)= & \frac{\sqrt{2} \cdot 3 \cdot \mu_{0} \cdot w_{n} \cdot I_{1}}{\delta \cdot \pi} \\
& \sum_{v}^{ \pm \infty} \frac{\xi_{n}(v) \xi_{s}(v)}{v} \cdot \cos \left(v \frac{\pi}{\tau_{s}} x-\omega t\right)
\end{aligned}
$$

\section{2) Mover field}

The exciter field induction $B_{\text {er }}$ caused by the permanent magnets is mapped on the basis of the geometry illustrated in Fig. 6 by a Fourier series approach

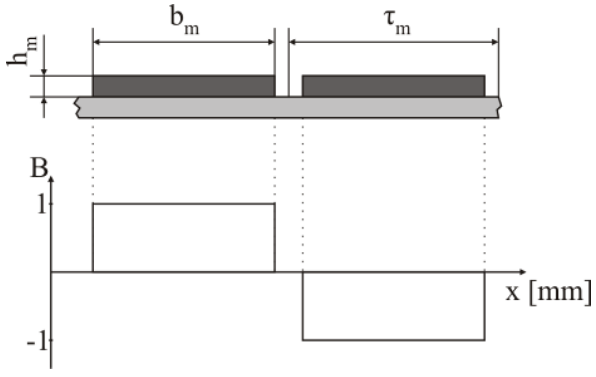

Figure 6.. Geometrical approach of the air gap induction caused by the permanent magnets

and results in (12).

$$
B_{e r}(x)=B_{0} \cdot \frac{4}{\pi} \sum_{v=1}^{\infty}\left[\begin{array}{l}
\xi_{s c h r}(v) \cdot \frac{1}{2 v+1} \\
\cdot \cos \left((2 v+1) \pi\left(\frac{1}{2}-\frac{b_{m}}{2 \tau_{s}}\right)\right) \\
\cdot \sin \left((2 v+1) \cdot\left(\frac{\pi}{\tau_{m}}(x)\right)\right)
\end{array}\right]
$$

The factor $\xi_{\text {schr }}(v)$ used from [7][8] contemplates the magnets mounted around the circumference of the generator, at an angle $\gamma_{\mathrm{n}}$. This arrangement has the benefit that the linear generator does not show any latching forces in operation.

$$
\xi_{\text {schr }}(v)=\frac{\sin \left(v \frac{\gamma_{n}}{2}\right)}{v \frac{\gamma_{n}}{2}}
$$

\section{3) Superposed field}

The resultant flux density is

$$
\begin{aligned}
& B(x, t)= B_{e r}(x)+B_{s}(x, t) \\
&= B_{0} \cdot \frac{4}{\pi} \sum_{v=1}^{\infty} \frac{1}{2 v+1} \cdot \cos \left((2 v+1) \pi\left(\frac{1}{2}-\frac{b_{m}}{2 \tau_{s}}\right)\right) \\
& \cdot \sin \left((2 v+1) \cdot\left(\frac{\pi}{\tau_{m}}(x)\right)\right) \\
&+ \frac{\sqrt{2} \cdot 3 \cdot \mu_{0} \cdot w_{n} \cdot I_{1}}{\delta \cdot \pi} \sum_{v}^{+\infty} \frac{\xi_{n}(v) \xi_{s}(v) \xi_{s c h r}}{v} \\
& \cdot \cos \left(v \frac{\pi}{\tau_{s}} x-\omega t\right)
\end{aligned}
$$

In Fig. 7 the exemplary result for the flux density is pictured. It is calculated with the material and geometrical parameters from [11]. 


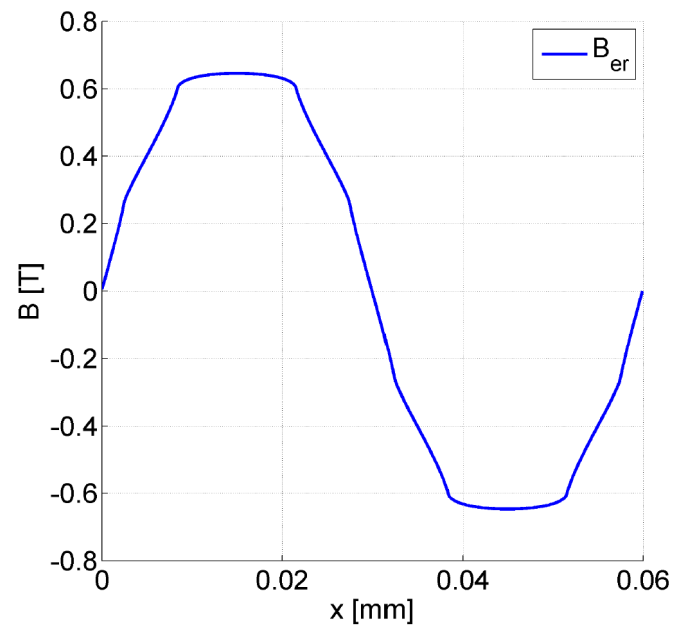

Figure 7. The superposition of the flux density from the stator and the mover

The results of the calculated iron losses are discussed together with the measured results in the chapter comparison.

\section{MEASUREMENT}

To answer the question how exact the calculation of the iron losses is, a prototype of the linear generator is developed and implemented. To realize the measurement a generally new test bench is built.

\section{A. Measurement environment}

A hydraulically-powered test bench is developed and constructed (see Fig. 8). With its model-based control the hydraulic cylinder built into the test bench replaces the combustion chamber and the gas spring. The linear generator component can thus be tested and measured without having to construct the remainder of the free-piston linear generator system.

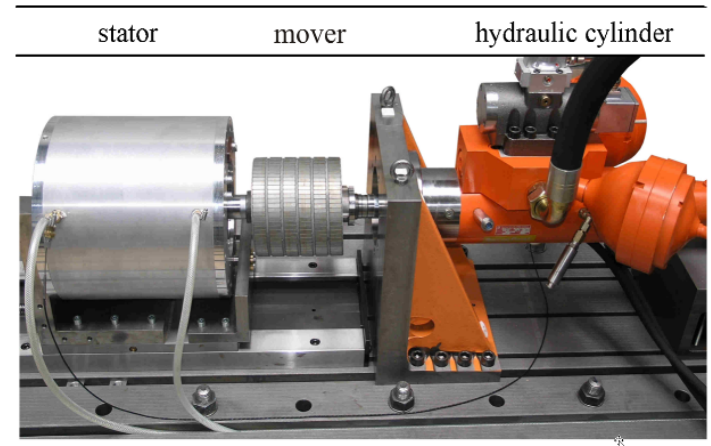

Figure 8. Installed linear generator with hydraulic cylinder

\section{B. Measuring method}

It is not possible to measure the iron losses directly, because the measured force includes the friction in the bearings and the inertial force. Based on (15)

$$
F_{f e}(f, h)=F_{\text {mess }}(f, h)-F_{\text {in }}(f, h)-F_{f r}(f, h)
$$

the friction based force $F_{\text {fr }}$ and the inertial force $F_{\text {in }}$ have to be measured first. For this a nonmagnetic mover is built up and placed in the linear generator to simulate the features of the mover which lead to the forces $F_{\text {fr }}$ and $F_{\text {in }}$.

With (16) the measured force can be converted into the energy.

$$
W_{l f e}=\int_{0}^{h} F_{l f e} d x
$$

The measurement of the energy $\left(\mathrm{W}_{\text {mess }}\right)$ generated from the friction, the inertial force and the iron losses are pictured in Fig. 9. In this graph the interpolation (interp) of the measured results is also shown.

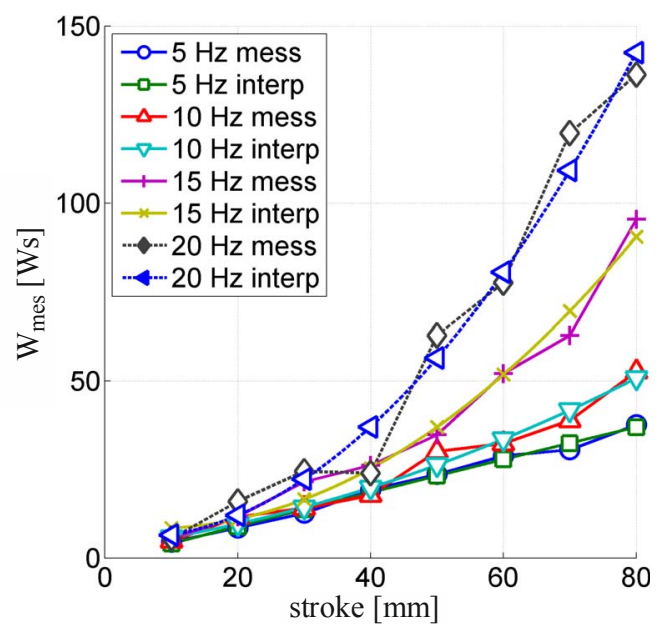

Figure 9. The sum of the measured friction and iron losses based force

The iron losses can be calculated with (15) by subtracting the friction losses $\mathrm{W}_{\text {lfr }}$ from the measured losses $\mathrm{W}_{\text {mess. }}$. The result is shown in Fig. 10.

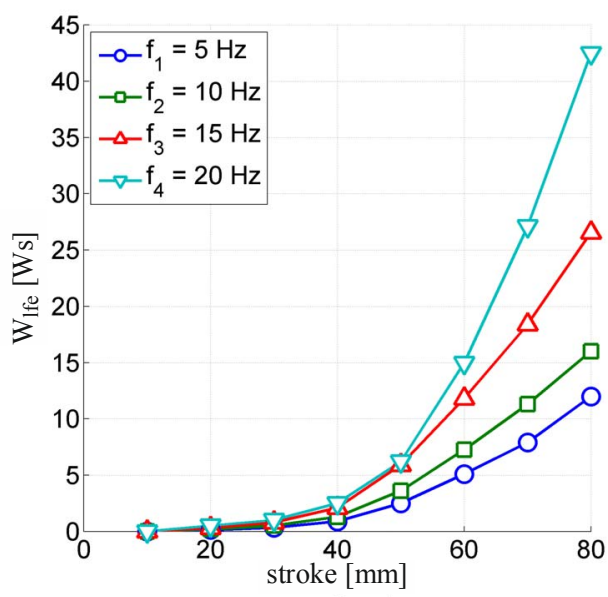

Figure 10. The measured and interpolated iron losses of the lineargenerator 
In the following graph the characteristic line of the calculated $\mathrm{W}_{\text {lfe,cal }}$ and the measured $\mathrm{W}_{\text {lfe,mess }}$ iron losses are pictured. The operating frequency is $15 \mathrm{~Hz}$.

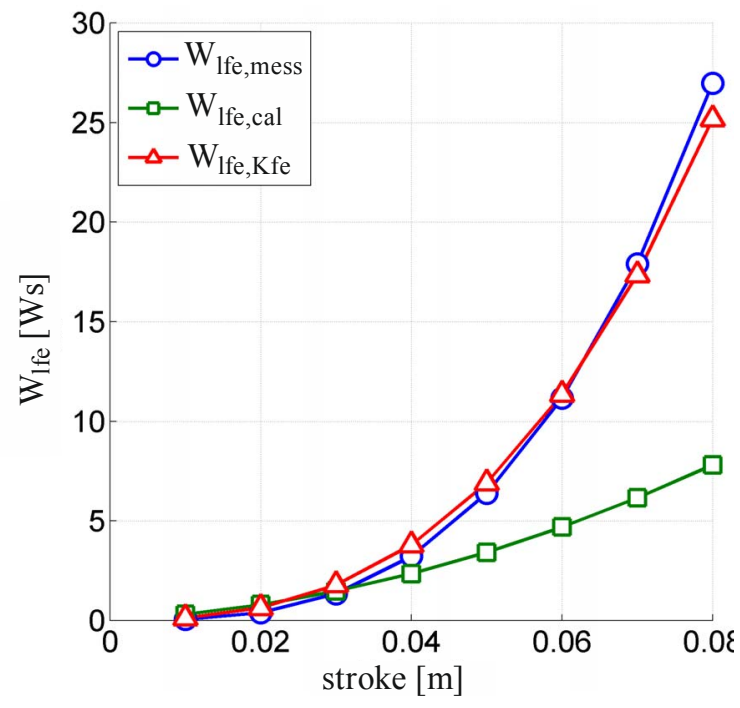

Figure 11. Calculated and measured iron losses

It is evident that the calculated losses are very different from the measured. And this happens even through the effects of the harmonics in the stator are included in the calculation.

The effect which creates this difference is the influence of the stroke on the frequency $f_{\text {eff. }}$ It is a specialty of a linear generator with a load point dependent stroke that the iron losses depend on the stroke of the mover.

If the effective frequency is calculated with the following equation

$$
f_{\text {eff }}=\frac{\text { stroke }}{\tau_{p}} \cdot f_{\text {mech }}
$$

the result is the characteristic line $\mathrm{W}_{\text {lfe, Kfe }}$ in Fig. 11. The line deviates from the measured losses very little.

\section{CONCLUSION}

The question if the iron losses from a linear generator can be calculated analytically and how exact they can be calculated is answered in this paper. The answer is that the iron losses of a special linear generator intended for use in a free piston linear generator can be calculated very accurate. To get these exact results it is important to include the losses of the flux density harmonics. It is also essential to calculate the effective frequency of the flux density as introduced in this publication for the first time. The analytical calculation of the linear generator and its iron losses is very well suited to the design of linear generators of this and similar topologies.

\section{REFERENCES}

[1] B. Metz, O. R. Davidson, P. R. Bosch, R. Dave and L. A. Meyer. "Climate Change 2007: Mitigation of Climate Change". Working Group III to the Fourth Assessment Report of the Intergovernmental Panel on Climate Change IPCC., Cambridge, Cambridge University Press, 2007.

[2] M. Gräf, S.-E. Pohl, F. Rinderknecht, C. Ferrari, "Free Piston Linear Generator with high partload efficency," in Proc. 2007 SAE Conf.

[3] S.-E. Pohl, "Der Freikolbenlineargenerator- Theoretische Betrachtung des Gesamtsystems und experimentelle Untersuchungen zum Teilsystem der Gasfeder" (The free-piston linear generator - theoretical consideration of the complete system and experimental studies in the subsystem of the gas spring). Ph.D. dissertation, Deutsches Zentrum für Luft und Raumfahrt, 2007.

[4] B. Ponick, G. Müller, K. Vogt, Berechnung elektrischer Maschinen (Calculation of electrical machines), vol.6. Weinheim: WILEY-VCH, 2008, p. 471

[5] H.-G. Herzog, "Grundzüge der elektromechanischen Energiewandlung," (Principles of the electromechanical conversion of energy), Fachgebiet Energiewandlungstechnik, Technische Universität München, München, Germany, WS 2006/2007.

[6] B. Ponick, G. Müller, K. Vogt, Berechnung elektrischer Maschinen (Calculation of electrical machines), vol.6. Weinheim: WILEY-VCH, 2008, p. 86,83

[7] W. Schuisky, Berechnung elektrischer Maschinen, München: SpringerVerlag, 1960.

[8] G. Müller, Theorie elektrischer Maschine, Winheim: WILEY-VCH, 1995.

[9] P. Vachenauer, K. Meyberg, Höhere Mathematik 2 (Higher mathematics 2). Berlin: Springer-Verlag, 1991.

[10] B. Ponick, G. Müller, K. Vogt, Grundlagen elektrischer Maschinen (Basics of electrical machines), vol.6. Weinheim: WILEY-VCH, 2006

[11] F. Rinderknecht and H.-G. Herzog "Adaptation and optimization of a linear generator for a hybrid vehicle concept," in Proc. Symposium EVS 25, Chen Zhen, China, 2010. 